\title{
Robust Performance Analysis for a Range of Frequencies
}

\author{
S.Boersma ${ }^{1}$, A.Korniienko ${ }^{2}$, K.Laib ${ }^{3}$, J.W.van Wingerden ${ }^{4}$
}

\begin{abstract}
Time domain specifications such as overshoot, rise time and tracking behaviour can be extracted from an amplitude frequency response. For uncertain systems we use for this an upper bound on the maximum amplitude frequency response. There are tools which can compute this upper bound for each frequency in a grid. Computing this upper bound can be computational expensive when studying a large scale system hence it is interesting to have a low dense frequency grid. However, in such a case, it can for example occur that the maximum peak of the amplitude frequency response occurs at a frequency which is not in this grid. A consequence is that the overshoot will not be determined well for the system. In this paper we will present a method such that this can not occur. We will augment the uncertainty set with an additional uncertain parameter. This uncertain parameter will cover the frequencies which are not covered by the grid. This allows us to do a robustness analysis for a range of frequencies. In this case we are sure that we do not miss any crucial information with respect to the amplitude frequency response lying in between the frequencies in the grid. We illustrate this using two simulation examples.
\end{abstract}

\section{INTRODUCTION}

It is possible to extract time domain specifications as e.g. overshoot, rise time and tracking behaviour from the amplitude frequency response of a system. The slope in the low frequency regions can give us for example information about the tracking behaviour of the system, the cross over frequency can give us information on the speed of the system and the maximum peak of the frequency response can give us information on the overshoot of the system. However, when dealing with uncertain systems as defined in for example [1] and [2], it is not sufficient to study one amplitude frequency response to extract this kind of information since the system is then a function of an infinite set containing the uncertainties. Hence, in order to make statements on the previously mentioned time domain specifications while looking at the amplitude frequency response, it is necessary to find the maximum among these responses. By using the latter we can guarantee that we for example will find the maximum peak of the frequency response among all the uncertainties in the set. This maximum amplitude frequency response can then be used to make statements on e.g. the overshoot. Due to the fact that the set containing the uncertainties is

${ }^{1}$ S.Boersma is with the Department DCSC, Delft University of Technology, Delft, The Netherlands s.boersma@tudelft.nl

${ }^{2}$ A.Korniienko is with the Laboratoire Ampère, Ecole Centrale de Lyon, Ecully, France anton.korniienko@ec-lyon.fr

${ }^{3}$ K.Laib is with the Laboratoire Ampère, Ecole Centrale de Lyon, Ecully, France khaled. laibedoctorant.ec-lyon. fr

${ }^{4}$ J.W.van Wingerden is with the Department DCSC, Delft University of Technology, Delft, The Netherlands J.W. vanWingerden@tudelft.nl infinite, it is from a practical point of view difficult to find the maximum amplitude frequency response. It is however possible to compute, per frequency, an upper bound on this maximum amplitude frequency response with the aid of $\mu$ analysis tools and corresponding convex optimization. We can then make statements on the time domain specifications using this upper bound.

As stated before, with $\mu$-analysis tools we can compute an upper bound on the maximum amplification of an uncertain system. The method has been studied well over the past few years. See for example [3] and [4]. In this paper we exploit $\mu$-analysis tools in order to solve a problem which can occur when applying it in the standard way. The problem is that when using $\mu$-analysis tools, the analysis is done only for specific frequencies in a grid. We do not analyse the intermediate frequencies hence we can not say anything about the maximum amplification of the system for these intermediate frequencies. It is in addition not always interesting to increase the density of the frequency grid since computing an upper bound is computational expensive. In this paper we introduce a method which allows us to also ensure the maximum amplification through the system for these intermediate frequencies while using the standard $\mu$ analysis tools. In other words, the method allows us to do a robustness analysis for a range of frequencies. The method can be applied to systems with multiple inputs and multiple outputs (MIMO) as well as single input single output (SISO) systems.

In [5], the authors propose a method to solve a similar problem. They suggest to test a frequency dependent infinite linear matrix inequality (LMI) conditions in the form of frequency independent finite LMI conditions. The latter conditions directly include the information about a specified frequency range to which they are applied. The method proposed in this paper is different. Instead of using "special LMI conditions", we represent the frequency as an uncertain parameter and then build our conditions based on the augmented system. The advantage is that we can use the traditional $\mu$-analysis tools which implies that people familiar with these techniques are able to directly apply the novel method proposed in this paper. The outcome will then not be an upper bound on the maximum amplification through an uncertain system for only one specific frequency but for a range of frequencies. The magnitude of this symmetric range then depends on the size of uncertainty we put on the frequency under consideration. In [6], the authors also propose to make the frequency an uncertain parameter and then use classical $\mu$-analysis tools to make statements on the system. To be more precise, a bound on the maximum 
amount of uncertainty is found for which the system is robustly stable.

In this paper we will however analyse if the system has a robust performance property instead of stability. The definition of robust performance in this paper will in addition differ from how it is defined in for example [1] and [2] and the final result will be generalised. Moreover, we illustrate the effectiveness of the approach using two examples. The first example will be a relatively simple second order system and serves to illustrate the method. The second example will be a large scale system where we, in addition to our method, will use the Hierarchical Approach [7].

This paper is structured as follows. In Section II, we introduce the necessary preliminary mathematical tools used throughout this paper. In Section III, we will give the problem formulation using an example. This example will illustrate the necessity of the novel method presented in this paper. In Section IV, we will introduce the novel method which will solve the problem as formulated in Section III. We will revisit the example and will illustrate the benefit of the new method while applying it on the same example. In Section $\mathrm{V}$ we will demonstrate the novel method on a large scale system. Since computing an upper bound on the amplitude frequency of such a high dimensional system is computational expensive, the Hierarchical Approach is used. The interested reader is referred to [7], [8] and [9] for more detailed information on the Hierarchical Approach.

\section{PRELIMINARIES}

In this section we summarise the theoretical background used in this paper. We begin by giving the considered system interconnection followed by a definition of robust performance and a theorem with which we can ensure this robust performance. Figure 1 presents the system interconnection under consideration. We define $z \in \mathbb{C}^{n_{z}}$ and $w \in \mathbb{C}^{n_{w}}$

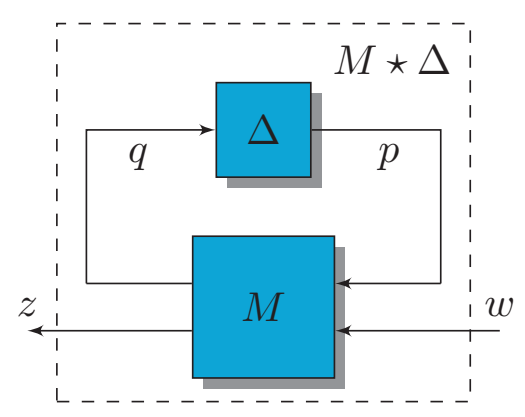

Fig. 1. The $M \Delta$-structure.

as the performance and exogenous input signal respectively. The signals $p \in \mathbb{C}^{n_{p}}$ and $q \in \mathbb{C}^{n_{q}}$ are the output and input respectively of the uncertainty block $\Delta \in \mathbb{R}^{n_{p} \times n_{q}}$. The transfer $M \star \Delta$ is the performance transfer function which we would like to analyse with stable $M \in \mathbb{C}^{n_{z}+n_{q} \times n_{w}+n_{p}}$ having the following state space representation:

$$
\begin{aligned}
\dot{x} & =A x+\left[\begin{array}{ll}
B_{p} & B_{w}
\end{array}\right]\left(\begin{array}{l}
p \\
w
\end{array}\right) \\
\left(\begin{array}{l}
q \\
z
\end{array}\right) & =\left[\begin{array}{l}
C_{q} \\
C_{z}
\end{array}\right] x+\left[\begin{array}{ll}
D_{q p} & D_{q w} \\
D_{z p} & D_{z w}
\end{array}\right]\left(\begin{array}{c}
p \\
w
\end{array}\right)
\end{aligned}
$$

The signal $z^{*}$ is the complex conjugate transpose of $z$ and the operator $\star$ is defined as the Redheffer star product [10]. In [1] this is referred to as the upper linear fractional transformation. We furthermore have the infinite uncertainty set $\boldsymbol{\Delta}=$ $\left\{\Delta \in \mathbb{R}^{n_{p} \times n_{q}} \mid \Delta=\operatorname{diag}\left(\delta_{1} I_{n_{1}}, \ldots, \delta_{r} I_{n_{r}}\right),\|\Delta\|_{\infty} \leq 1\right\}$ with real scalar uncertainties $\delta_{k} \in \mathbb{R}$, for $k=1, \ldots, r$ with $r$ the number of uncertainties and $n_{k}$ the number of repetitions of the uncertainty $\delta_{k}$ and $n=\sum_{k=1}^{r} n_{k}$, the size of the uncertainty $\Delta$. We note that the work presented in this paper can be extended to a more general class of uncertainties e.g. we could include complex uncertainties in the set $\boldsymbol{\Delta}$. This is however not necessary for illustrating the novel method hence we only consider real uncertainties in the set $\boldsymbol{\Delta}$. We define the following definition of robust performance. Note that this definition is different from the one used in e.g. [1] and [2].

Definition 1 (Robust Performance): If for a specific frequency $\omega_{i}$ there exists a $\gamma \in \mathbb{R}$ such that, for $M$ and $\Delta$ evaluated at the frequency $\omega_{i}$, the LMI

$$
\left(\begin{array}{c}
M \star \Delta \\
I
\end{array}\right)^{*}\left[\begin{array}{cc}
I & 0 \\
0 & -\gamma^{2} I
\end{array}\right]\left(\begin{array}{c}
M \star \Delta \\
I
\end{array}\right)<0 \quad \forall \Delta \in \Delta
$$

holds, robust performance is ensured for the $M \Delta$-structure with performance transfer $M \star \Delta$ at the frequency $\omega_{i}$.

When minimising $\gamma^{2}$, the best possible robust performance can be found. Note that in the single input single output (SISO) case, the minimum $\gamma$ is the maximum amplitude frequency response of the performance transfer $M \star \Delta$ among all $\Delta \in \Delta$ for the frequency $\omega_{i}$. In the multiple input multiple output (MIMO) case, this will be the maximum singular value representing the maximum amplification through the performance transfer $M \star \Delta$ among all $\Delta \in \Delta$ for the frequency $\omega_{i}$. The problem of testing if robust performance is satisfied is that it should hold for all $\Delta \in \Delta$ with $\Delta$ being an infinite set. In other words, we try to find a maximum frequency response among an infinite number of frequency responses when considering the SISO case. The following theorem is taken from [8] and provides us with tools to ensure, for a specific frequency $\omega_{i}$, robust performance as defined in Definition 1.

Theorem 1 (Robust Performance Theorem): Robust performance as defined in Definition 1 is ensured for a specific frequency $\omega_{i}$ if and only if there exists a matrix $\Phi_{\Delta}$ with partitions $X_{\Delta}, Y_{\Delta}$ and $Z_{\Delta}$ such that

$$
\left(\begin{array}{c}
\Delta \\
I
\end{array}\right)^{*} \underbrace{\left[\begin{array}{cc}
X_{\Delta} & Y_{\Delta} \\
Y_{\Delta}^{*} & Z_{\Delta}
\end{array}\right]}_{\Phi_{\Delta}}\left(\begin{array}{c}
\Delta \\
I
\end{array}\right) \geq 0 \quad \forall \Delta \in \Delta
$$




$$
\left(\begin{array}{c}
I \\
M
\end{array}\right)^{*}\left[\begin{array}{cc|cc}
X_{\Delta} & 0 & Y_{\Delta} & 0 \\
0 & -\gamma^{2} I & 0 & 0 \\
\hline Y_{\Delta}^{*} & 0 & Z_{\Delta} & 0 \\
0 & 0 & 0 & I
\end{array}\right]\left(\begin{array}{c}
I \\
M
\end{array}\right)<0
$$

hold.

As stated in Theorem 1, two LMIs should be verified to ensure robust performance where the one given in (3) depends on $\Delta$ and should hold for all $\Delta \in \Delta$. It is shown in [11] that by choosing a parametrisation of the matrix $\Phi_{\Delta}$, the LMI in (3) is always ensured. A consequence of introducing such a parametrisation is that the "if and only if" condition to ensure robust performance as given in Theorem 1 will become an "if" condition. This is stated in the following corollary.

Corollary 1: Let the matrix $\Phi_{\Delta}$ belong to a bounded set $\Phi_{\Delta}$ such that (3) is always satisfied. Then robust performance as given in Definition 1 is ensured for a specific frequency $\omega_{i}$ if there exists a Hermitian matrix $\Phi_{\Delta} \in \boldsymbol{\Phi}_{\boldsymbol{\Delta}}$ with partitions $X_{\Delta}=X_{\Delta}^{*}, Y_{\Delta}, Z_{\Delta}=Z_{\Delta}^{*} \geq 0$ and $X_{\Delta} \in \mathbb{C}^{n_{p} \times n_{p}}$ and $Z_{\Delta} \in \mathbb{C}^{n_{q} \times n_{q}}$ such that (4) holds.

One possible parametrisation is the DG-scaling [11] and appropriate for the set of uncertainties we take into consideration in this paper. The interested reader is referred to [12] for other parametrisations. The DG-scaling is defined as the set:

$$
\begin{gathered}
\boldsymbol{\Phi}_{\Delta}=\left\{\Phi_{\Delta} \mid \Phi_{\Delta}=\left[\begin{array}{cc}
-D & G \\
G^{*} & D
\end{array}\right]\right\} \\
\text { with } \quad D=\operatorname{bdiag}\left(D_{1}, \ldots, D_{r}\right) \\
\text { and } \quad G=\operatorname{bdiag}\left(G_{1}, \cdots, G_{r}\right)
\end{gathered}
$$

And we have that $D_{k}=D_{k}^{*}>0 \in \mathbb{C}^{n_{k} \times n_{k}}$ with $D$ having the property $D \Delta=\Delta D, G_{k}=-G_{k}^{*} \in \mathbb{C}^{n_{k} \times n_{k}}$ for $k=1, \ldots, r$. We note that if one wants to take a more general uncertainty set into account, i.e. include also complex uncertainty, the $G$ matrix in (5) has to be redefined as e.g. given in [11]. We omit this here since it does not contribute to the demonstration of the new method presented in this paper.

Suppose now that we are given a nominally stable uncertain system $M \star \Delta$ with $\Delta \in \Delta$. The objective is to find the maximum amplitude frequency response among all $\Delta \in \Delta$ for each frequency in the grid. Finding such a maximum response is from a practical point of view not interesting since the set $\boldsymbol{\Delta}$ is infinite as discussed previously. It is however possible to compute, for each frequency in a grid, an upper bound on the maximum amplitude frequency response based on Corollary 1 and the DG-scaling given in (5). This upper bound can be found by solving for each frequency $\omega_{i}$ in the grid the following problem:

$$
\begin{array}{ll}
\min _{\gamma^{2}, D, G} & \gamma^{2} \\
\text { s.t. } & \left(\begin{array}{c}
I \\
M
\end{array}\right)^{*}\left[\begin{array}{cc|cc}
-D & 0 & G & 0 \\
0 & -\gamma^{2} I & 0 & 0 \\
\hline G^{*} & 0 & D & 0 \\
0 & 0 & 0 & I
\end{array}\right]\left(\begin{array}{c}
I \\
M
\end{array}\right)<0
\end{array}
$$

With $D$ and $G$ as defined in (5). The value $\gamma^{\text {opt }}=$ $\sqrt{\arg \min \gamma^{2}}$ is then the upper bound on the maximum amplitude frequency response for the frequency $\omega_{i}$. If we solve the problem given in (6) for the grid $\omega=\left[\omega_{1}, \omega_{2}, \ldots, \omega_{N}\right]$ we get an upper bound for each of these frequencies. This upper bound can give us then information about previously discussed time domain performance specifications, the original objective. Now that we have given all the necessary mathematical tools, we can proceed by giving a problem which can occur when using these tools to compute an upper bound and to make statements on time domain specifications by using this upper bound.

\section{Problem Formulation}

Since the problem given in (6) is a frequency dependent problem, an upper bound for only the frequencies in the grid under consideration can be ensured. Hence we do not know what happens in between the subsequent frequencies in the grid. This implies that it is possible, for example, that we do not detect the maximum peak of the amplitude frequency response with, as a consequence, a guarantee of time domain specifications other than the system actually exhibits. Indeed, the more dense we make the frequency grid, the more likely it is that we do not miss any important information. However, for high dimensional systems, it can be computationally expensive to compute the upper bound hence it is interesting to have a non dense frequency grid. We will illustrate a possible problem which can occur when applying the classical method to a relatively simple system in the following subsection.

\section{A. Numerical Example}

Given the following nominally stable uncertain SISO system

$$
M \star \Delta=\frac{1}{m s^{2}+b s+k}
$$

with $b=b_{0}\left(1+W_{b} \delta_{b}\right)$ and $k=k_{0}\left(1+W_{k} \delta_{k}\right)$ with $\left|\delta_{b}\right| \leq$ $1,\left|\delta_{k}\right| \leq 1, \Delta=\operatorname{diag}\left(\delta_{b}, \delta_{k}\right)$, i.e we have that $n_{z}=n_{w}=$ $1, n_{q}=n_{p}=2$. We furthermore have that $m=10, b_{0}=$ $.3, k_{0}=10, W_{b}=.25 b_{0}$ and $W_{k}=.05 k_{0}$. We can define $M$ accordingly as

$$
M=\left(\begin{array}{ccc}
-W_{b} s & -W_{b} s & W_{b} s \\
-W_{k} & -W_{k} & W_{k} \\
-1 & -1 & 1
\end{array}\right) \frac{1}{m s^{2}+b_{0} s+k_{0}}
$$

Then, for the system given in (7), we can solve the problem given in (6) for a frequency grid $\omega$ i.e. we can compute an upper bound on the maximum frequency response among all $\Delta \in \Delta$ for the frequencies in the grid. In Figure 2 we depict the results including the frequency response of the nominal model. The latter is considered as continuous to illustrate the possible problem which can arise using standard $\mu$ analysis tools. When using the upper bound to, for example, make statements on the maximum peak of the frequency response, we obtain a maximum of 6.91 [dB]. However, the nominal model already has a maximum of 10.46 [dB] though on a different frequency. This frequency is however not in the set $\omega$ used for computing the upper bound. Hence, 
when using the upper bound, we will guarantee time domain specifications which the system actually does not exhibits.

It could thus be interesting to be able to guarantee an upper bound for the complete grid $\omega$, i.e. also for the intermediate frequencies. We will present in the following subsection a method which allows us to do this. We will see that we need to solve problem (6) with modified $\Delta$ and $M$ for a grid of frequencies and that we are able to ensure an upper bound for each frequency in the grid and for a small range around each of the frequencies. The proposed method will be illustrated using the same numerical example as presented in this section.

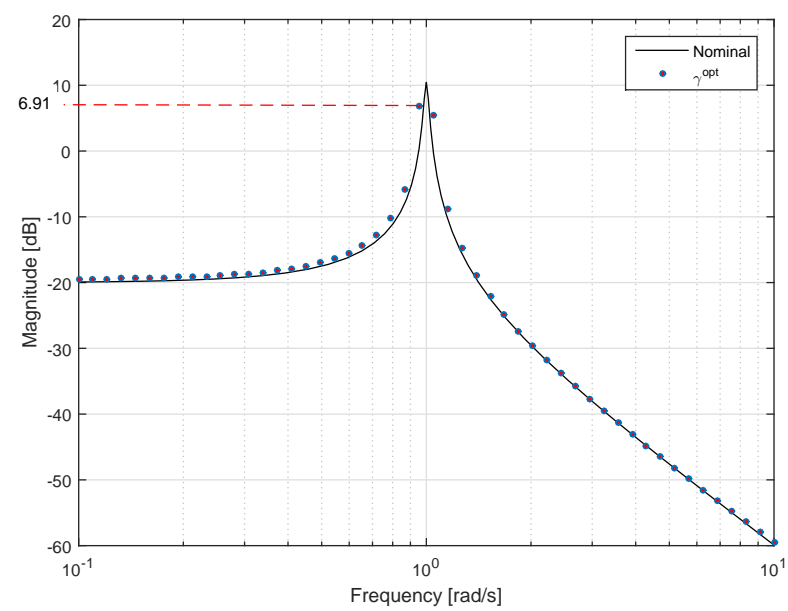

Fig. 2. Frequency response of the nominal model (black line) and the upper bound on the maximum frequency response.

\section{Proposed Method}

In order to ensure an upper bound for a range around a frequency we introduce an additionally uncertain parameter $\omega_{i}=\omega_{i}^{0}\left(1+W_{\omega} \delta_{\omega}\right)$ with $\left|\delta_{\omega}\right| \leq 1$. In other words, we make each frequency in the grid an uncertain parameter with $\omega_{i}^{0}$ being one nominal frequency out of the grid and $W_{\omega}$ defining the (symmetric) range around this nominal frequency $\omega_{i}^{0}$. A consequence is that we will be able to guarantee an upper bound on the maximum amplitude frequency response for the nominal frequency $\omega_{i}^{0}$ and also for a symmetric range with amplitude defined by $W_{\omega}$ around the frequency $\omega_{i}^{0}$. Note that in this new case, the frequency grid under consideration will then be a grid with nominal frequencies defined as $\omega=\left[\omega_{1}^{0}, \omega_{2}^{0}, \ldots, \omega_{N}^{0}\right]$. Then each of these frequencies in the grid can be considered as a nominal frequency and for each of these frequencies, the upper bound can be computed, i.e. the problem given in (6) can be solved. In order to practically clarify the proposed method, we present in Figure 3 the integrator block in the original situation (left) and in the situation when the frequency is an uncertain parameter (right). Indeed, if we close the loop of the block scheme on the right and let $s \rightarrow i \omega_{i}^{0}$ we get the transfer:

$$
\frac{1}{i \omega_{i}^{0}\left(1+W_{\omega} \delta_{\omega}\right)}, \quad\left|\delta_{\omega}\right| \leq 1
$$

After the replacement of all the integrator blocks in our system, we can define an augmented uncertainty block

$$
\Delta_{a}=\operatorname{diag}\left(\Delta, I_{n} \delta_{\omega}\right), \quad\left\|\Delta_{a}\right\|_{\infty} \leq 1
$$

with $n$ the number of integrators in the system under consideration. Then we can compute an augmented $M_{a}$ matrix accordingly. Note that the augmented uncertainty block $\Delta_{a}$ will always contain one additional $n$ times repeated uncertainty block. It is possible to generalise the above and

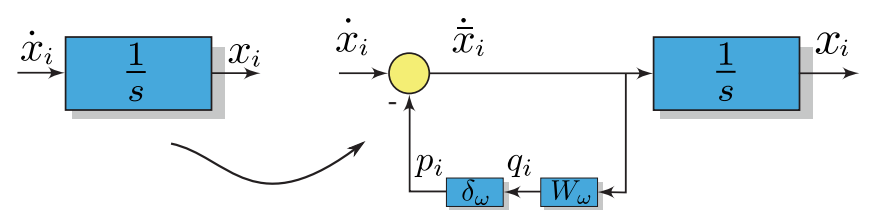

Fig. 3. Integrator block (left) and integrator block with uncertain frequency (right).

depict the augmented uncertainty $\Delta_{a}$ and $M_{a}$ in the $M \Delta$ structure. This is illustrated in Figure 4. The partitions in the matrix $M_{a}$ are given in (1). Now that we have explained

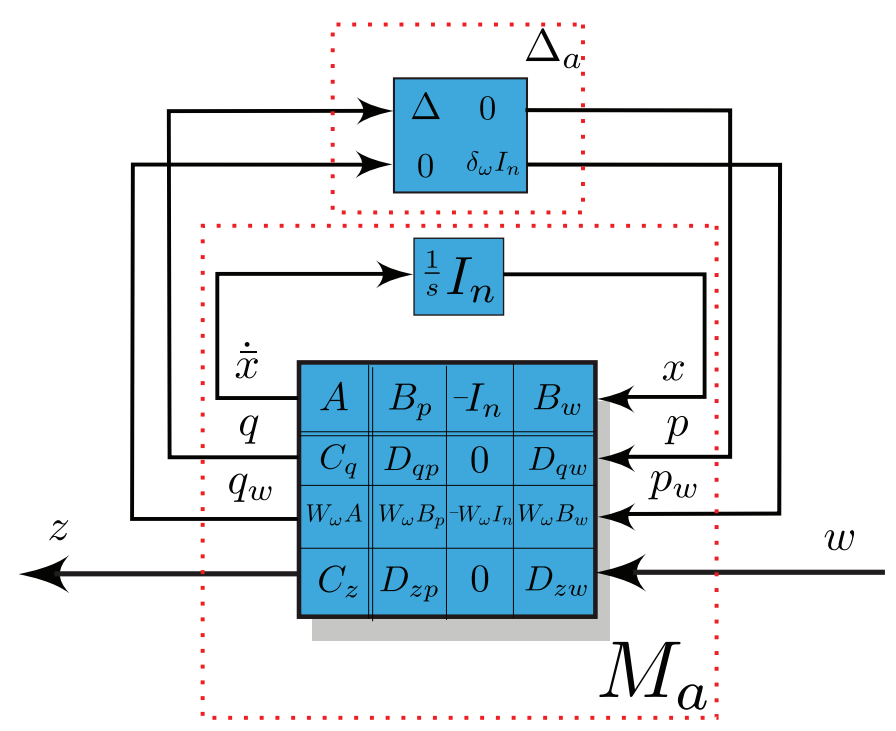

Fig. 4. The $M \Delta$-structure with augmented uncertainty $\Delta_{a}$ and matrix $M_{a}$.

the necessary steps to ensure an upper bound for a range of frequencies and gave the definitions of the augmented uncertainty $\Delta_{a}$ and $M_{a}$, we are ready to give the following theorem.

Theorem 2 (RP Theorem for a range of frequencies): Robust performance as defined in Definition 1 is ensured for a nominal frequency $\omega_{i}^{0}$ and a symmetric frequency 
range with magnitude $\left|W_{\omega}\right|$ around $\omega_{i}^{0}$ if we solve:

$$
\begin{array}{ll}
\min _{\gamma^{2}, D, G} & \gamma^{2} \\
\text { s.t. } & \left(\begin{array}{c}
I \\
M_{a}
\end{array}\right)^{*}\left[\begin{array}{cc|cc}
-D & 0 & G & 0 \\
0 & -\gamma^{2} I & 0 & 0 \\
\hline G^{*} & 0 & D & 0 \\
0 & 0 & 0 & I
\end{array}\right]\left(\begin{array}{c}
I \\
M_{a}
\end{array}\right)<0
\end{array}
$$

with $D$ and $G$ as defined in (5) according to the new uncertainty block $\Delta_{a}$ as given in (10). The value $\gamma^{\text {opt }}=$ $\sqrt{\operatorname{argmin} \gamma^{2}}$ is then the upper bound on the maximum frequency response for the frequency $\omega_{i}^{0}$ and a symmetric frequency range with magnitude $\left|W_{\omega}\right|$ around $\omega_{i}^{0}$.

The proof of this theorem follows directly from Theorem 1 and Corollary 1. If we solve the problem as defined in (11) for the grid $\omega=\left[\omega_{1}^{0}, \omega_{2}^{0}, \ldots, \omega_{N}^{0}\right]$ we get an upper bound for each of these frequencies and a range around each of these frequencies. This upper bound can then give us information about time domain performance specifications, the original objective. Observe that the range for which we can ensure robust performance depends on $\left|W_{\omega}\right|$ hence the latter should be chosen such that each subsequent frequency will be overlapped by its neighbours. In the following subsection, we will give the same numerical example as we presented before though now having uncertainty on the frequency as discussed in this subsection.

\section{A. Numerical Example}

In this section we reconsider again the system as given in (7). We will now make the frequency an uncertain parameter as we have discussed in the previous subsection. Since the system in (7) has two integrators, the new uncertain system will have one two times repeated uncertain parameter in addition to the two uncertain parameters $b$ and $k$. The new uncertainty block is defined as $\Delta_{a}=\operatorname{bdiag}\left(\delta_{b}, \delta_{k}, \delta_{\omega} I_{2}\right)$ (hence $n_{p}=n_{q}=4$ ) and the new $M_{a}$ matrix can then be defined accordingly as:

$$
\begin{aligned}
& M_{a}= \\
& \left(\begin{array}{ccccc}
-W_{b} s & -W_{b} s & -W_{b} m s i & -W_{b} k_{0} i & W_{b} s \\
-W_{k} & -W_{k} & -W_{k} m i & -W_{k}\left(m s+b_{0}\right) i & W_{k} \\
-W_{\omega} s & -W_{\omega} s & -W_{\omega} m s i & W_{\omega} k_{0} i & W_{\omega} s \\
-W_{\omega} & -W_{\omega} & -W_{\omega} m i & -W_{\omega}\left(m s+b_{0}\right) i & W_{\omega} \\
-1 & -1 & -m i & -\left(m s+b_{0}\right) i & 1
\end{array}\right) \frac{1}{m s^{2}+b_{0} s+k_{0}}
\end{aligned}
$$

When solving the problem as given in (11) while using appropriate matrices $D$ and $G$ as given in (5), we obtain, after properly choosing $W_{\omega}$, the (continuous) upper bound as illustrated in Figure 5. Note that we use the same frequency grid as we did when computing the upper bound as depicted in Figure 2. However, due to the fact that we made the frequency an uncertain parameter, we are now also able to guarantee an upper bound in between the frequencies under consideration. It can be seen that we guarantee a maximum peak of $13.18[\mathrm{~dB}]$ and that the nominal frequency response is situated below the computed upper bound. It should be clear that the upper bound is a bound on the maximum amplitude frequency response hence we can conclude that the maximum amplification of the system is not more than
$13.18[\mathrm{~dB}]$. Using the previous method we guaranteed an maximum amplification of 6.91 [dB] which was already violated by the nominal model. This implies that by using the new method, we able to ensure time domain specifications of the system which it really exhibits. The example given in

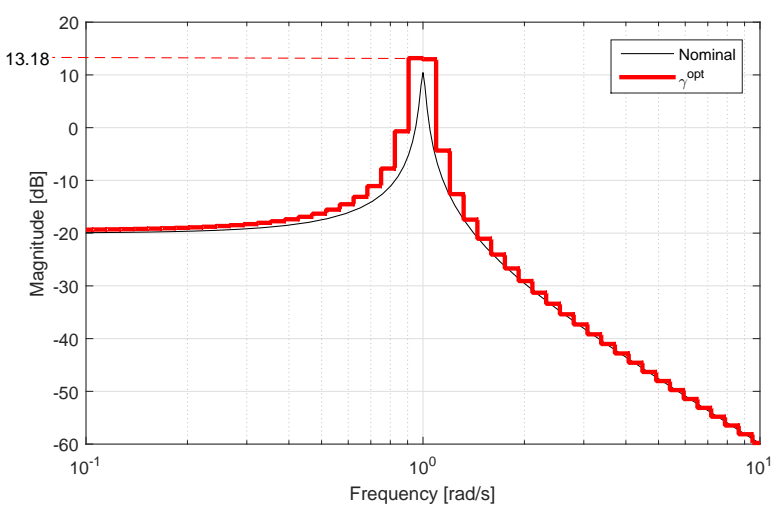

Fig. 5. Frequency response of the nominal system (back line) and the upper bound on the maximum frequency response.

this section illustrates that, by using the proposed method, we are able to ensure time domain specifications of a system which are guaranteed not violated. By using the standard technique we can guarantee a better performance due to a "wrong" choice of the frequency grid while this performance is not exhibited by the system under consideration. The novel method only needs a proper choice of the weight $W_{\omega}$ such that the subsequent frequencies overlap. For the example considered until now, we could argue to increase the density of the grid $\omega$ to solve the frequency gridding problem because we are dealing with a relatively simple system. Then we will most likely not miss the peak in the maximum amplitude frequency response. However, when dealing with high dimensional systems, increasing the density of $\omega$ is not always interesting. This is due to the fact that computing an upper bound for high dimensional systems are computational expensive. In the following section we apply the presented method on such a high dimensional system.

\section{Large Scale Network Application}

The large scale system studied in this section will be a network of $N=16$ phase locked loops (PLLs) and is taken from [13]. Such a network can for example be used to distribute and synchronise a clock signal in a multicore processor [13]. The network can be presented using the $M \Delta$-structure as depicted in Figure 6. Here we have the static matrix $\tilde{M}$ which defines how the PLLs influence each other in order to synchronize the network [7] (see Equation (13)) and $\tilde{\Delta}=\operatorname{diag}\left(T^{1}, T^{2}, \ldots, T^{16}\right)$ with $T^{l}$ defined as one PLL in the network. We furthermore have the SISO performance transfer $\tilde{M} \star \tilde{\Delta}$ as the global transfer function for which we would like to compute an upper bound, i.e. for which we would like to ensure the optimal robust performance as defined in Definition 1. This example 


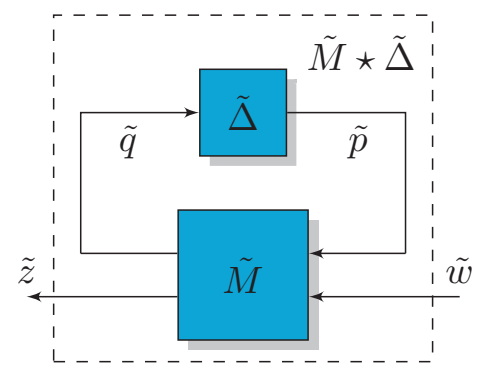

Fig. 6. Block scheme representation of the large scale system.

is suitable for illustration since the performance is naturally evaluated in the frequency domain [13].

\section{A. PLL network description}

In the network considered in this paper, all the PLLs are homogeneous and their individual uncertainty blocks belong to the same uncertainty set $\boldsymbol{\Delta}$. These uncertainties "capture" the technological dispersions which occur due to the manufacturing process. They can be presented as parametric uncertainties belonging to the same set $\Delta$. Then, the description of the $N$ PLLs is:

$$
T^{l}\left(i \omega_{i}^{0}\right)=\frac{k_{l}\left(i \omega_{i}^{0}+a_{l}\right)}{-\left(\omega_{i}^{0}\right)^{2}+k_{l} i \omega_{i}^{0}+k_{l} a_{l}}, \quad \forall l \in\{1, \ldots, N\}
$$

where $k_{l} \in[0.76,6.84] \times 10^{4}, a_{l} \in[91.1,273.3]$ and $\omega_{i}^{0}$ is the current frequency. Furthermore, $T^{l}\left(i \omega_{i}^{0}\right)$ can written as the interconnection of a certain and an uncertain part:

$$
T^{l}\left(i \omega_{i}^{0}\right)=\Delta^{l} \star M^{\mathrm{PLL}}, \quad \Delta^{l} \in \boldsymbol{\Delta}
$$

with $\Delta^{l}$ denoted as:

$$
\boldsymbol{\Delta}=\left\{\Delta^{l} \in \mathbb{R}^{2 \times 2} \mid \Delta^{l}=\operatorname{diag}\left(\delta_{k_{l}}, \delta_{a_{l}}\right)\right\}
$$

\section{B. Performance analysis}

The performance analysis of this network consists in computing an upper bound on the maximum amplitude frequency response. The problem of using standard $\mu$-analysis tools on such high dimensional system is the computation effort needed to compute an upper bound on the maximum amplitude frequency response due to the large scale aspect. In order to reduce this effort, the authors in [7], [8] and [9] use a method called the Hierarchical Approach initially proposed in [14]. This method allows to reduce the computational effort with respect to the time necessary when using standard techniques. Nevertheless, it is interesting to reduce the resolution of the frequency grid for which we compute the upper bound in both methods: direct and hierarchical. Indeed, the denser the frequency grid, the higher the computation effort. However, we can miss important information if we reduce the density and as a consequence, make false statements on the time performance of the system as we have seen in the numerical example presented in Section III. Therefore, it is interesting to apply our method on the network of PLLs using the Hierarchical Approach.

\section{Hierarchical Approach for a range of frequencies}

For the network considered here, the Hierarchical Approach consists of two steps, the local and the global step. The final goal is to compute an upper bound on the maximum amplitude frequency response of the SISO performance transfer $\tilde{M} \star \tilde{\Delta}$, i.e. we would like to ensure the optimal robust performance as defined in Definition 1 for the performance transfer. In order to do so we can use Theorem 1. We can unfortunately not use the parametrisation as defined in (5) since in that case, the LMI in (3) is not by definition verified. Hence the first (local) step of the Hierarchical Approach consists of finding a suitable parametrisation of the set $\boldsymbol{\Phi}_{\boldsymbol{\Delta}}$ such that (3) always holds. We will, in the following subsections, describe the steps done in the Hierarchical Approach in more detail.

1) Local step: In this step we make the frequency in each PLL uncertain such that we obtain $\Delta_{a}^{l}=$ $\operatorname{diag}\left(\delta_{k_{l}}, \delta_{a_{l}}, \delta_{\omega} I_{2}\right)$. We then define $T_{a}^{l}$ accordingly which we can write as $T_{a}$ due to the fact that the PLLs are homogeneous. Now we are interested in characterizing the input-output behaviour of each PLL using integral quadratic constraints (IQCs) which can, in the complex plane, be interpreted by simple geometric forms: disc [7], band [8] and cone [9] such that for each frequency $\omega_{i}^{0}$ we have that:

$$
\left(\begin{array}{c}
T_{a} \\
I
\end{array}\right)^{*}\left[\begin{array}{cc}
X_{k} & Y_{k} \\
Y_{k}^{*} & Z_{k}
\end{array}\right]\left(\begin{array}{c}
T_{a} \\
I
\end{array}\right)<0
$$

with $k \in\{$ disc,band,cone $\}$. Details concerning the formulation of the IQCs are in [7], [8] and [9]. Finding the matrices $X_{k}, Y_{k}$ and $Z_{k}$ is also referred to as finding a suitable embedding which can be characterised by IQCs. We can apply Theorem 2 to obtain these IQCs for a range of frequencies around $\omega_{i}^{0}$.

2) Global step: The different IQCs obtained in the local step can be used to characterize the 16 PLLs gathered in $\tilde{\Delta}$ such that:

$$
\left(\begin{array}{c}
\tilde{\Delta} \\
I
\end{array}\right)^{*}\left[\begin{array}{cc}
X & Y \\
Y^{*} & Z
\end{array}\right]\left(\begin{array}{c}
\tilde{\Delta} \\
I
\end{array}\right) \geq 0
$$

holds with:

$$
\begin{aligned}
& X=-\operatorname{diag}\left(\sum_{k} \tau_{1 k} X_{k}, \ldots, \sum_{k} \tau_{N k} X_{k}\right) \\
& Y=-\operatorname{diag}\left(\sum_{k} \tau_{1 k} Y_{k}, \ldots, \sum_{k} \tau_{N k} Y_{k}\right) \\
& Z=-\operatorname{diag}\left(\sum_{k} \tau_{1 k} Z_{k}, \ldots, \sum_{k} \tau_{N k} Z_{k}\right)
\end{aligned}
$$

where $k \in\{$ disc, band,cone $\}$ and $\tau_{i k}>0$ are introduced to increase the number of decision variables in the global step which can reduce the possible conservatism.

Now we can apply Theorem 2 to solve the problem of finding an upper bound on the maximum amplitude global transfer frequency response by solving for each frequency 
$\omega_{i}^{0}$ in the grid the following problem:

$$
\begin{array}{ll}
\min _{\gamma^{2}, X, Y, Z} & \gamma^{2} \\
\text { s.t. } & \left(\begin{array}{c}
I \\
\tilde{M}
\end{array}\right)^{*}\left[\begin{array}{cc|cc}
-X & 0 & -Y & 0 \\
0 & -\gamma^{2} I & 0 & 0 \\
\hline-Y^{*} & 0 & -Z & 0 \\
0 & 0 & 0 & I
\end{array}\right]\left(\begin{array}{c}
I \\
\tilde{M}
\end{array}\right)<0
\end{array}
$$

The value $\gamma^{\text {opt }}=\sqrt{\arg \min \gamma^{2}}$ is then the upper bound on the maximum amplitude frequency response of the transfer $\tilde{M} \star \tilde{\Delta}$ for the frequency $\omega_{i}^{0}$ and a range around this frequency defined by $W_{\omega}$. Note that the problem defined in (12) is an adapted version of the problem defined (6). When we solve the problem given in (12) for the grid $\omega=\left[\omega_{1}^{0}, \omega_{2}^{0}, \ldots, \omega_{N}^{0}\right]$, the (continuous) upper bound as depicted in Figure 7 is obtained. We observe in Figure 7 that

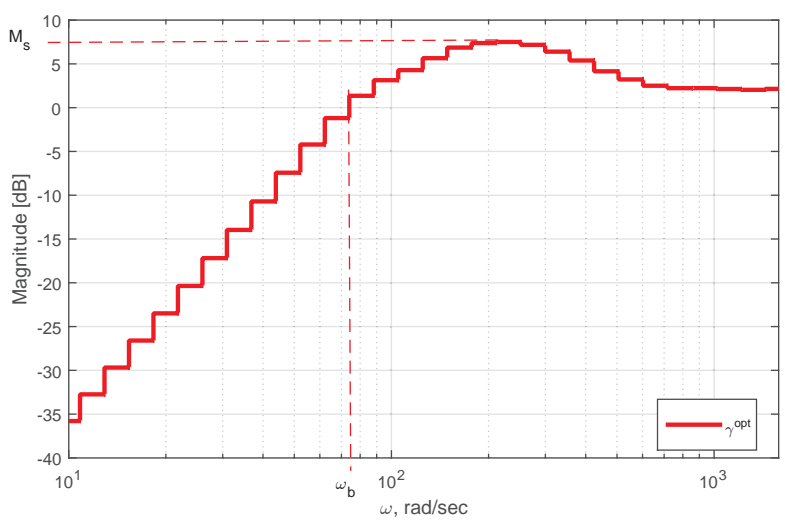

Fig. 7. The upper bound on the maximum frequency response of the performance transfer function.

the performance transfer can be seen as a sensitivity function from which bandwidth $\omega_{b}$, maximum peak $M_{s}$ and slope in the low frequency region can be extracted. These can give us information on the time domain specifications rise time, overshoot and tracking behaviour of the system respectively. Due to the fact that we use the proposed method, we are able to ensure a continuous upper bound and will for sure not miss any important information about the system.

\section{DISCUSSION}

In this paper we presented a method which allows us to compute an upper bound for a range of frequencies on the maximum amplitude frequency response of a system. The range depend on the weight $W_{\omega}$. If the latter is chosen such that the subsequent frequencies in the frequency grid overlap, then we are able to guarantee an continuous upper bound on the maximum amplitude frequency response from the first frequency in the grid to the last. It should be noted that the solution can become conservative. The source of this conservatism is the additional uncertainty on the frequency one introduces in the system using the new method. This conservatism depends on $\left|W_{\omega}\right|$ which on its turn depends on the resolution of the frequency grid. The denser the frequency grid, the smaller we can make $\left|W_{\omega}\right|$ since we need to set this such that there is an overlap between the subsequent frequencies. The upper bound will on its turn then become less conservative.

\section{CONCLUSIONS}

The method presented in this paper allows us to ensure an upper bound on the maximum amplitude frequency response for a range of frequencies. This could be interesting according to the example given in Section III. Here we have illustrated that, by using a relatively simple SISO example, information on the time domain specifications can be ensured though these specifications are not really exhibited by the system. This overestimation occurred sine we are not able to, using classical techniques in the frequency domain, ensure an upper bound for a range of frequencies. The method in this paper however allows us to do so.

We also have showed that the new method can be applied to a more advanced high dimensional system. It is even more interesting to do so since in such a case it is more important to keep the density of the frequency low due to the otherwise increasing computational load. So the proposed method allows us to keep the density of the frequency grid low while also being able to ensure a continuous upper bound from the first to the last frequency in the grid. By doing so we are sure that the time domain specifications guaranteed using the upper bound are the specifications the systems actually has.

\section{REFERENCES}

[1] S. Skogestad and I. Postlethwaite, Multivariable feedback control analysis and design. John Wiley and Sons Chischester, 2005.

[2] K. Zhou, J. C. Doyle, and K. Glover, Robust and Optimal Control. Prentice-Hall, Inc., 1996.

[3] J. C. Doyle, "Structured uncertainty in control system design," IEEE Conference on Decision and Control, pp. 260-265, 1985.

[4] M. K. H. Fan, A. L. Tits, and J. C. Doyle, "Robustness in the presence of mixed parametric uncertainty and unmodeled dynamics," IEEE Transactions on Automatic Control, vol. 63, no. 1, pp. 25-38, 1991.

[5] T. Iwasaki and S. Hara, "Generalized KYP lemma: unified frequency domain inequalities with design applications," IEEE Transactions on Automatic Control, vol. 50, no. 1, pp. 41-59, 2005.

[6] G. Ferreres and J.-M. Biannic, "A $\mu$ analysis technique without frequency gridding," Proceedings of the 1998 American Control Conference, vol. 4, pp. 2294-2298, June 1998.

[7] M. Dinh, A. Korniienko, and G. Scorletti, "Embedding of uncertainty propagation: application to hierarchical performance analysis," IFAC Symposium on System, Structure and Control, vol. 5, no. 1, pp. 190195, 2013.

[8] M. Dinh, A. Korniienko, and G. Scorletti, "Convex hierarchicalrchical analysis for the performance of uncertain large scale systems," IEEE Conference on Decision and Control, pp. 5979- 5984, 2014.

[9] K. Laib, A. Korniienko, G. Scorletti, and F. Morel, "Phase IQC for the hierarchical performance analysis of uncertain large scale systems," IEEE Conference on Decision and Control (to appear), 2015.

[10] J. Doyle, A. Packard, and K. Zhou, "Review of LFTs, LMIs, and $\mu$," Proceedings of the 30th IEEE Conference on Decision and Control, vol. 2, pp. 1221- 1232, 1991.

[11] C. Scherer and S. Weiland, Linear Matrix Inequalities in Control. Lecture Notes, Delft Center for Systems and Control (DCSC), Delft University of Technology, The Netherlands.

[12] G. Scorletti, X. Bombois, M. Barenthin, and V. Fromion, "Improved efficient analysis for systems with uncertain parameters," IEEE Conference on Decision and Control, pp. 5038-5043, 2007.

[13] A. Korniienko, G. Scorletti, E. Colinet, E. Blanco, J. Juillard, and D. Galayko., "Control law synthesis for distributed multi-agent systems: Application to active clock distribution networks," IEEE American Control Conference, pp. 4691-4696, 2011. 
[14] M. G. Safonov, "Propagation of conic model uncertainty in hierarchical systems," IEEE Transactions on Automatic Control, vol. 28, no. 6, pp. 701-709, 1983. 